\title{
Editorial: Refractory Astrochemistry
}

\author{
Ryan C. Fortenberry ${ }^{1 *}$ and Stefan T. Bromley ${ }^{2,3}$ \\ ${ }^{1}$ Department of Chemistry and Biochemistry, University of Mississippi, Oxford, MS, United States, ${ }^{2}$ Departament de Ciència de \\ Materials i Química Física and Institut de Química Teòrica i Computatcional (IQTCUB), Universitat de Barcelona, Barcelona, Spain, \\ ${ }^{3}$ Institució Catalana de Recerca i Estudis Avançats (ICREA), Passeig L/uis Companys 23, Barcelona, Spain
}

Keywords: oxygen, silicates, carbides, dust grains, astrochemistry, spectroscopy

\section{Editorial on the Research Topic}

\section{Refractory Astrochemistry}

The cornerstone of all chemistry (and astrochemistry is no exception) has long-been the element carbon with its capacity to form numerous strong bonds to itself and other atoms. Although observation has verified the presence of carbonaceous and organic species in space, other materials also play an important role in astrochemistry. Although, over $90 \%$ of all the unique molecules observed thus far in the Universe contain element- 6 in volatile, organic materials, carbon also forms refractory carbides in C-rich circumstellar environments. Additionally, in carbon poor environments the chemistry of oxygen becomes dominant.

OPEN ACCESS

Edited by: Ankan Das,

Indian Centre for Space Physics, India

Reviewed by:

Marzio Rosi,

University of Perugia, Italy Albert Rimola,

Universitat Autònoma de Barcelona, Spain Mattia Melosso, University of Bologna, Italy

${ }^{*}$ Correspondence: Ryan C. Fortenberry r410@olemiss.edu

Specialty section: This article was submitted to Astrochemistry, a section of the journal Frontiers in Astronomy and Space Sciences

Received: 20 January 2022 Accepted: 10 February 2022 Published: 25 February 2022

Citation:

Fortenberry RC and Bromley ST (2022) Editorial: Refractory Astrochemistry. Front. Astron. Space Sci. 9:859101. doi: 10.3389/fspas.2022.859101
The Earth is a prime example inclusive of the lithosphere, atmosphere, magnetosphere, and beyond. Nearly all of these telluric regions are dominated, or at least strongly influenced, by oxygen and not carbon. In fact, oxygen is the most abundant element in the Universe behind only hydrogen and helium. The detailed chemistry of this element and its refractory oxides has often been neglected in astronomical studies. This special issue is an attempt to highlight the exciting and creative work going on in the inorganic realm of refractory astrochemistry whether carbon is included or not.

Refractory materials, as opposed to volatiles, most simply are those that do not easily enter the gas phase. They need extremely high temperatures (typically $>1000 \mathrm{~K}$ ) to do so. Often such materials are viewed as ceramics, glasses, or crystals. However, all elements' nuclei smaller than iron are believed to be formed in the cores of massive stars making the pathways to any of these types of materials often long and arduous. This special issue will touch on some of the ways that inorganic processes (even inorganic processes involving carbon) can take place to provide a rich depth of astrochemistry that is not reliant upon volatile, complex organic molecules.

This issue has contributions that showcase chemical reactions of carbon monoxide on imperfect surfaces of silicate (i.e., Si-based oxides; Fioroni and DeYonker) as well as ice chemistry taking place on porous silicate grains (Christianson and Garrod). Other contributed papers examine the growth of silicate dust grains from nanoscale precursors in the interstellar medium (Rimola and Bromley), and how such grains may be necessary to create the most abundant molecule in the Universe, $\mathrm{H}_{2}$ (Suhasaria and Mennella).

Beyond silicates, silicon can also combine with other elements for the creation of refractory carbide materials. Another theoretical study reports on the calculated ionization potentials of the smallest grains of SiC (Gobrecht). Small silver carbide species are also studied in a joint theoretical-experimental investigation, where they are found to promote the formation of Ag clusters and catalyzed hydrocarbon growth (Bérard et al.). Vibrational and rotational spectra of inorganic hydrogen-containing species are computed in another paper (Watrous et al.), which may be helpful in deducing their spectral fingerprints along the pathway from atoms to dust grains as observed by the newly launched James Webb Space Telescope. 
Despite the breadth of topics examined in this work, refractory astrochemistry is really in its infancy. A huge swathe of experimental and theoretical work is still open to chemical exploration. This research helps to bring astrochemistry in line with many aspects of geochemistry, and the two fields will likely collaborate closely in the future. Indeed, the same materials that are being made in interstellar environments also may have novel terrestrial applications. Hence, materials chemistry may find beneficial applications from the study of refractory astrochemistry especially in those applications where refractory surfaces may catalyze chemical reactions. In all things, the pursuit of new knowledge inevitably leads to new technology while also painting a clearer picture of the science originally motivating the study. The hope of this special issue is to continue to encourage practitioners of inorganic astrochemistry that their efforts are needed for filling out and adding to our astrophysical knowledge and to convince others that these research pursuits are giving unique and beneficial insights to astrochemistry, geochemistry, materials science, and beyond.

\section{AUTHOR CONTRIBUTIONS}

All authors listed have made a substantial, direct, and intellectual contribution to the work and approved it for publication.

Conflict of Interest: The authors declare that the research was conducted in the absence of any commercial or financial relationships that could be construed as a potential conflict of interest.

Publisher's Note: All claims expressed in this article are solely those of the authors and do not necessarily represent those of their affiliated organizations, or those of the publisher, the editors and the reviewers. Any product that may be evaluated in this article, or claim that may be made by its manufacturer, is not guaranteed or endorsed by the publisher.

Copyright (c) 2022 Fortenberry and Bromley. This is an open-access article distributed under the terms of the Creative Commons Attribution License (CC $B Y)$. The use, distribution or reproduction in other forums is permitted, provided the original author(s) and the copyright owner(s) are credited and that the original publication in this journal is cited, in accordance with accepted academic practice. No use, distribution or reproduction is permitted which does not comply with these terms. 